\title{
Melioidosis in New Caledonia: a dominant strain in a transmission hotspot
}

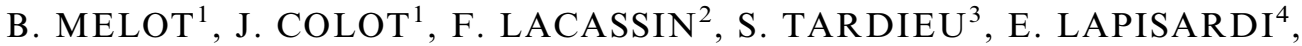 \\ M. MAYO ${ }^{5}$, E. P. PRICE ${ }^{5}$, D. S. SAROVICH ${ }^{5}$, B. J. CURRIE ${ }^{5}$ AND \\ C. GOARANT ${ }^{6 *}$ \\ ${ }^{1}$ Institut Pasteur in New Caledonia, Medical Microbiology Laboratory, Nouméa, New Caledonia \\ ${ }^{2}$ Centre Hospitalier Territorial de Nouvelle-Calédonie, Internal Medicine and Infectious Diseases, Nouméa, New \\ Caledonia \\ ${ }^{3}$ Centre Hospitalier du Nord de Nouvelle-Calédonie, Medical Microbiology Laboratory, Koumac, New Caledonia \\ ${ }^{4}$ Centre Hospitalier du Nord de Nouvelle-Calédonie, Emergency Unit and Acute Care Unit, Poindimié, New \\ Caledonia \\ ${ }^{5}$ Menzies School of Health Research, Charles Darwin University, Darwin, NT, Australia \\ ${ }^{6}$ Institut Pasteur in New Caledonia, Leptospirosis Research and Expertise Unit, Nouméa, New Caledonia
}

Received 3 August 2015; Final revision 21 October 2015; Accepted 22 October 2015;

first published online 6 November 2015

\section{SUMMARY}

Melioidosis is an infectious disease caused by Burkholderia pseudomallei, a bacterium endemic in Southeast Asia and northern Australia. In New Caledonia, sporadic cases were first described in 2005; since then, more cases have been identified. To improve our understanding of melioidosis epidemiology in New Caledonia, we compared the local cases and B. pseudomallei isolates with those from endemic areas. Nineteen melioidosis cases have been diagnosed in New Caledonia since 1999, mostly severe and with frequent bacteraemia, leading to three $(16 \%)$ fatalities. All but one occurred in the North Province. Besides sporadic cases caused by non-clonal strains, we also identified a hotspot of transmission related to a clonal group of $B$. pseudomallei that is phylogenetically related to Australian strains.

Key words: Epidemiology, melioidosis, molecular epidemiology.

\section{INTRODUCTION}

Melioidosis is a severe bacterial disease caused by the strict aerobic hydro-telluric Gram-negative bacterium Burkholderia pseudomallei. Infection occurs following percutaneous inoculation, inhalation or ingestion of contaminated water or soil. The disease is well recognized in animals (swine, horses, goats, sheep) as well as humans [1]. Human-to-human transmission is rare [2].

\footnotetext{
* Author for correspondence: Dr C. Goarant, Institut Pasteur de Nouvelle-Calédonie, BP 61, 98845 Nouméa Cedex, New Caledonia. (Email: cgoarant@pasteur.nc)
}

Melioidosis has highly polymorphic clinical presentations, primarily pneumonia or skin or internal abscesses [3]. This disease is endemic in Southeast Asia and northern Australia, where it causes 20\% and $32 \%$ of community-acquired bacteraemic pneumonia, respectively $[4,5]$. It is increasingly recognized as occurring in diverse locations which are mostly in the tropics [6], including Papua New Guinea (PNG) in the Melanesian region [7]. In northern Thailand and northern Australia, the melioidosis incidence rate is, on average, 20 cases $/ 100000$ per year, with similar rates reached in endemic regions of PNG [7]. Although most cases reported in Europe and the United States have been linked to infection contracted 
in endemic locations that become symptomatic upon return, endemicity occurs in some specific Western regions, as recently documented in Puerto Rico [8].

Melioidosis presentation can be overwhelming or asymptomatic. Cohort studies have identified risk factors for both exposure (e.g. contact with soil in rice farmers) and clinical expression of the infection (e.g. diabetes, hazardous alcohol use, chronic respiratory disease or renal impairment). Pulmonary involvement is the most frequent clinical presentation $(45-58 \%)$, and a major differential diagnosis of this form is pulmonary tuberculosis. Cutaneous forms are less frequent (13-17\%), and neurological involvement only occurs in a small proportion of cases $(3 \%$ in Thailand to $10 \%$ in Australia) [9]. Approximately $50 \%$ of patients are bacteraemic. The fatality rate is usually very high (10-50\% depending on regions and cohorts studied) [3].

B. pseudomallei was first identified in New Caledonian soil in 1984 [10]. Serological tests conducted on animals at that time showed previous exposure in $1 \cdot 2 \%(4 / 320)$ of cattle, $38 \%(46 / 120)$ of horses and 49\% (74/150) of swine but no B. pseudomallei could be cultured from animal abscesses. The first human case was confirmed in New Caledonia in 1999 [11]. Since then, nine other cases have been reported $[12,13]$. An additional nine cases have been bacteriologically confirmed in recent years. All but one lived in the North Province and none had history of travel abroad. The geographical pattern of melioidosis over time in New Caledonia led us to hypothesize a possible migration of the disease southwards on the North-east coast of New Caledonia in recent years.

\section{MATERIAL AND METHODS}

\section{Patients and medical files}

Melioidosis cases were diagnosed by the isolation of B. pseudomallei from the bloodstream $(n=14,74 \%)$ or a deep abscess $(n=6,32 \%)$ or both. Demographical, sociological and clinical data were retrieved from medical files. When useful and where possible, additional interviews were conducted directly with patients.

\section{Characterization and typing of New Caledonian B. pseudomallei isolates}

Bacterial species confirmation and antimicrobial susceptibility testing was made by API 20NE and by ATB PSE (bioMérieux, France).
Data for multilocus sequence typing (MLST; www. bpseudomallei.mlst.net) [14] were available for 13 isolates either from earlier work [13] or were extracted from whole genome sequencing data generated on the Illumina HiSeq2000 platform (Illumina Inc., USA) for this study. Sixteen strains from 19 cases were tested for $\operatorname{bim} A_{\mathrm{Bm} / \mathrm{Bp}}$ loci [15], and four-locus multilocus variable number tandem repeat analysis (MLVA-4) was also performed [16] to determine strain relatedness.

Concatenated MLST sequences were aligned with BioEdit [17], and a phylogenetic tree was built using the Phylo-Win program [18] that applied the Neighbour-Joining method and Kimura's two-parameter distances. Reliability of topologies was assessed by the bootstrap method with 500 replicates.

\section{RESULTS}

The geographical distribution of cases is summarized in Figure 1. All but one case occurred in the North Province, with a particular region of highest incidence in the area surrounding the village of Ponerihouen. The incidence in the affected districts of this particular region was as high as 6 cases $/ 100000$ annually. Interestingly, the only case from the South Province (in Paita) stayed in the village of Poindimié, on the northeast coast, for some days around New Year's Eve in 2008. This patient consulted at the Poindimié hospital for pneumonia in early January 2008, although melioidosis was confirmed only at the end of January, in Paita, South Province. The temporal pattern is further detailed in Figure 2.

The demographical, geographical and clinical data for the 19 melioidosis cases reviewed here are summarized in Table 1. Patients were mostly middle-aged (mean age 55 years) men (M/F sex ratio 3:2). Most were immunocompromised $(n=15,79 \%)$ due to diabetes $(n=4)$, chronic renal impairment $(n=3)$, alcoholism $(n=4)$, transplantation $(n=1)$, systemic lupus erythematosus $(n=1)$, cancer [mesothelioma $(n=1)$, or oesophageal cancer $(n=1)]$. In addition, four patients had chronic obstructive respiratory syndrome, including two with concomitant chronic alcoholism. All patients had either contact with horses, pigs or deer or direct contact with soil through chronic wounds $(21 \%, n=4)$.

We compared our data with two historical cohorts from Thailand and Australia and the Melanesian cohort of the Balimo region in PNG [7] (Table 1). Of the 19 New Caledonian cases, the most common 


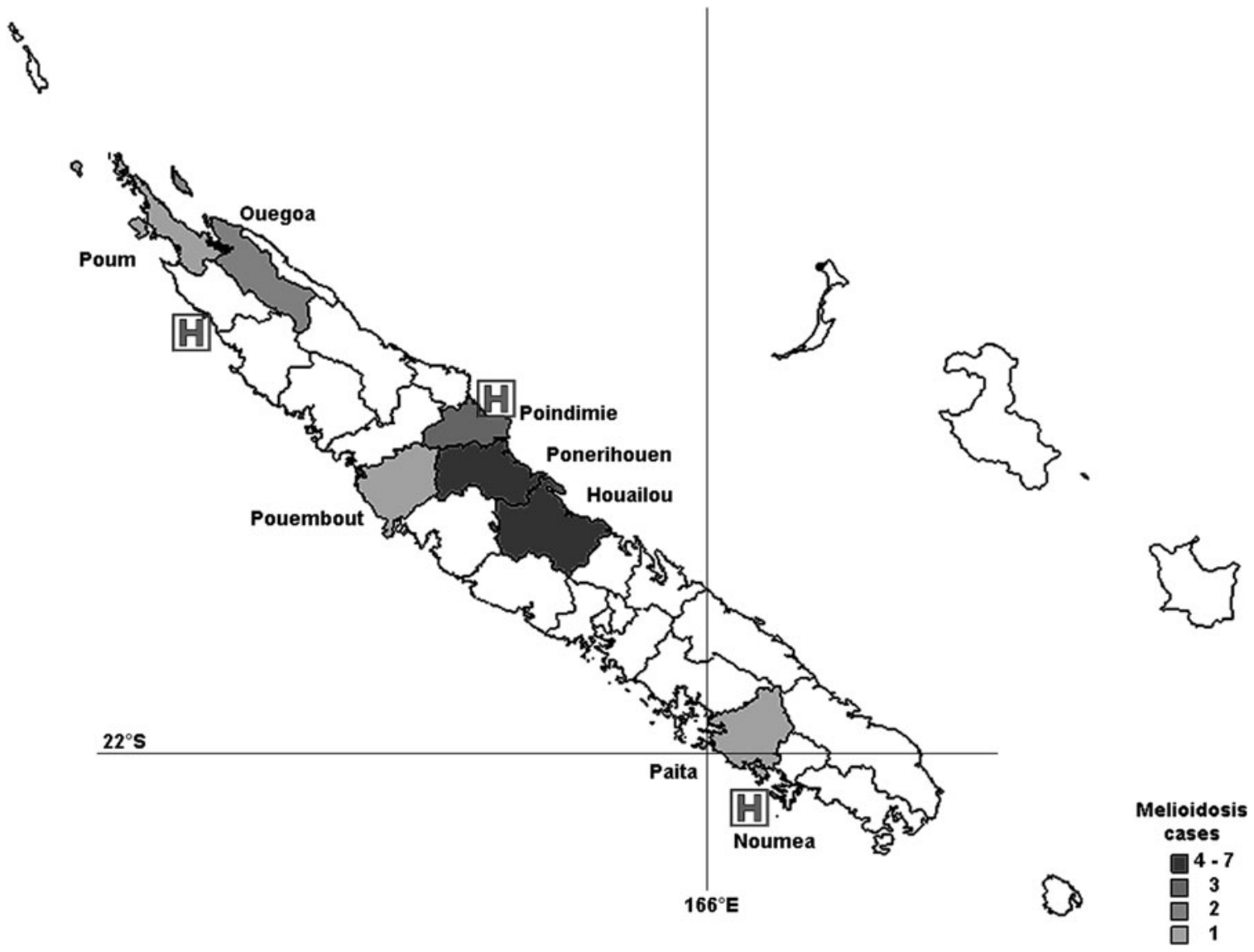

Fig. 1. Map of New Caledonia showing the capital (Nouméa), the villages mentioned in the text and the geographical distribution of the 19 melioidosis cases and the hospitals (large $\mathbf{H}$ signs). Note that there is evidence to suggest that the South Province case (Paita) was infected in Poindimié.
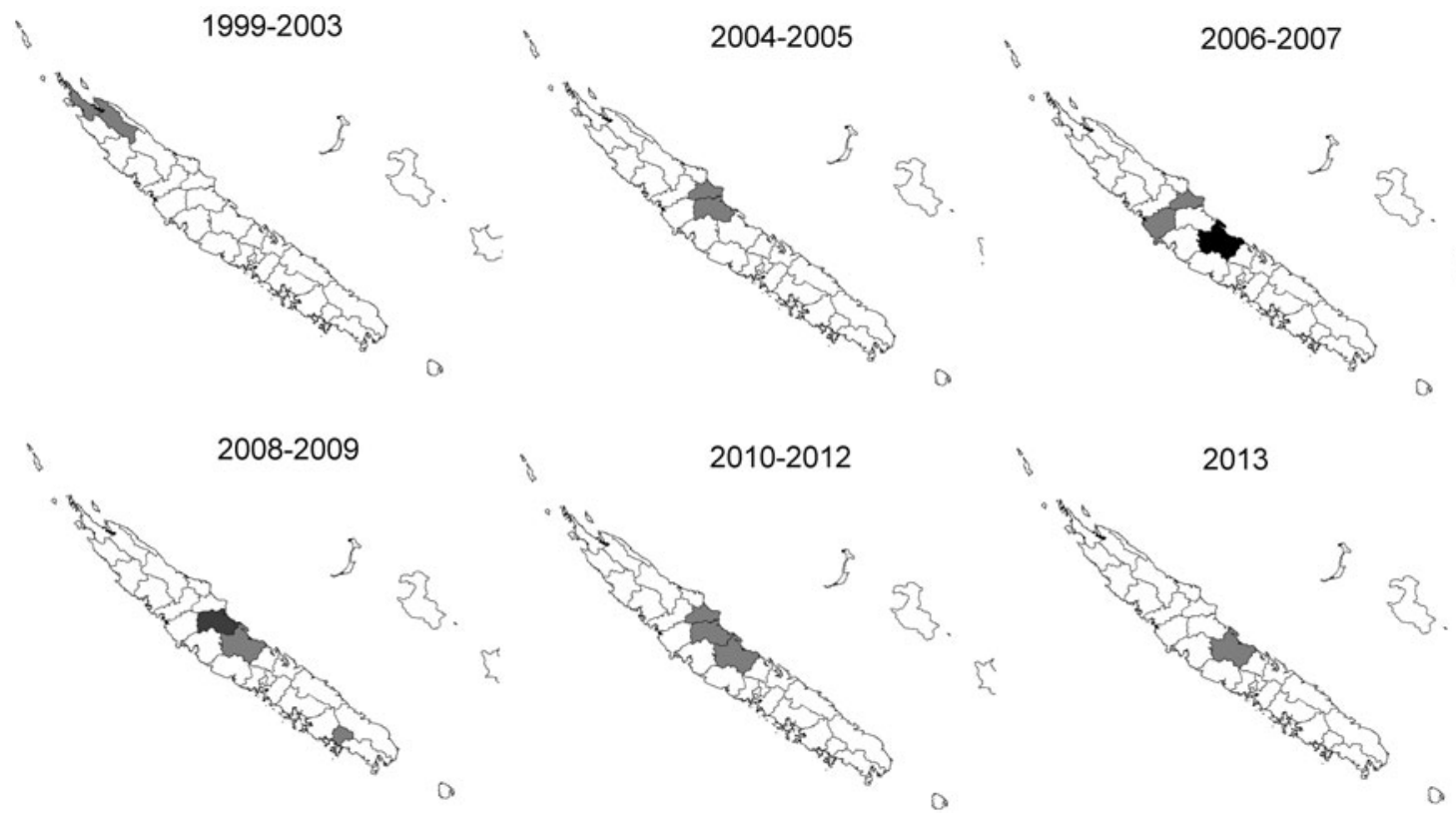

Fig. 2. Spatio-temporal distribution of melioidosis cases over the period 1999-2013. Note that there is evidence to suggest that the 2008 South Province case was acquired in Poindimié (North-east). 
Table 1. New Caledonian melioidosis cases and comparison with Thai and Australian historical cohorts

\begin{tabular}{|c|c|c|c|}
\hline & New Caledonia $(n=19)$ & $\begin{array}{l}\text { Thailand }(n=686) \\
\text { (cited in [3]) }\end{array}$ & Australia $(n=252)[2]$ \\
\hline Sex ratio $(M: F)$ & $3: 2$ & $3: 2$ & $3: 2$ \\
\hline Age (years) & 55 & 49 & $48 \cdot 5$ \\
\hline Risk factors & $\begin{array}{l}\text { Diabetes, alcohol, renal failure, } \\
\text { chronic lung disease }\end{array}$ & $\begin{array}{l}\text { Diabetes, rice } \\
\text { culture }\end{array}$ & $\begin{array}{l}\text { Diabetes, renal failure, alcohol, } \\
\text { kava }\end{array}$ \\
\hline Chronic & $32 \%^{\mathrm{a}}$ & $10 \%{ }^{\mathrm{a}}$ & $12 \%^{\mathrm{a}}$ \\
\hline Pneumonia & $53 \%$ & $45 \%$ & $58 \%$ \\
\hline $\begin{array}{l}\text { Meningitis/ } \\
\text { neurological }\end{array}$ & $5 \%$ & $3 \%$ & $4 \%$ \\
\hline Skin disease & $32 \%{ }^{b}$ & $13 \%{ }^{b}$ & $17 \%$ (primary and secondary) \\
\hline Bacteraemia & $74 \%{ }^{\mathrm{c}}$ & $58 \%$ & $46 \%{ }^{\mathrm{c}}$ \\
\hline Fatality & $16 \%{ }^{\mathrm{d}}$ & $40 \%{ }^{\mathrm{d}}$ & $20 \%$ \\
\hline
\end{tabular}

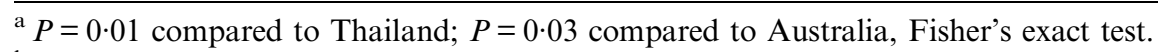

${ }^{\mathrm{b}} P=0.03$ compared to Thailand, Fisher's exact test.

${ }^{\mathrm{c}} P=0.03$ compared to Australia, Fisher's exact test.

${ }^{\mathrm{d}} P=0.03$ compared to Thailand, Fisher's exact test.

clinical form was pneumonia in $10(53 \%)$ patients, whereas six $(32 \%)$ had cutaneous melioidosis. Of note, one case of meningitis due to $B$. pseudomallei was diagnosed, and $74 \%(n=14)$ of patients had bacteremia. The disease was acute in $13(68 \%)$ cases and chronic in six $(32 \%)$; no cases were attributed to activation from latency, although for most of the patients it was not possible to precisely evaluate the date of initial infection. Four $(21 \%)$ patients were admitted to an intensive care unit. The overall case-fatality rate was $16 \%(n=3)$, which is similar to rates observed in northern Australia. One of the patients had leptospirosis one year before being diagnosed with melioidosis but none was diagnosed with any co-infection at the time of their melioidosis.

All strains had typical antibiotic resistance profiles [19], remaining susceptible to ceftazidime, imipenem, meropenem, doxycycline and amoxicillin-clavulanate. For six patients, treatment information was not available. During the intensive phase therapy, eight patients received ceftazidime, one patient received imipenem, and five patients received either sulfamethoxazole + trimethoprim, an unspecified third-generation cephalosporin or amoxicillin-clavulanate. Intravenous therapy ranged from 3 to 24 days duration (median 16.3 days).

MLST showed four different sequence types (STs) out of 13 isolates, with 10 ST292 isolates and single isolates of ST291, ST645 and ST1360 (Table 2). MLVA-4 further split the ST292 strains into MLVA-4 type $304(n=3)$, four single locus variants (SLVs) of MLVA-4 type 304 (MLVA-4 types 300,
Table 2. New Caledonian B. pseudomallei isolates described in this study: year and origin of the patients

\begin{tabular}{lcllc}
\hline \hline Isolate ID & Year & Location & $\begin{array}{l}\text { MLVA-4 } \\
\text { type }\end{array}$ & $\begin{array}{l}\text { MLST } \\
\text { type }\end{array}$ \\
\hline IPNC 336 & 1999 & Poum & 434 & 1360 \\
IPNC 91 & 2002 & Ouegoa & 299 & 291 \\
IPNC 269 & 2003 & Ponerihouen & 433 & 292 \\
IPNC 122 & 2004 & Poindimié & 300 & 292 \\
IPNC 140 & 2005 & Ponerihouen & 301 & 292 \\
IPNC 150 & 2006 & Poindimié & 302 & 292 \\
IPNC 158 & 2006 & Houailou & 303 & 292 \\
IPNC 149 & 2006 & Houailou & 304 & 292 \\
IPNC 445 & 2006 & Houailou & 304 & n.d. \\
IPNC 163 & 2007 & Houailou & 304 & 292 \\
IPNC 164 & 2008 & Pouembout & 305 & 645 \\
IPNC 221 & 2008 & Paita (but & 431 & 292 \\
& & probably & & \\
& & infected in & & \\
IPNC 238 & 2009 & Ponerihouen & 432 & 292 \\
IPNC 185 & 2009 & Houailou & 304 & 292 \\
IPNC 267 & 2012 & Houailou & 304 & n.d. \\
IPNC 389 & 2013 & Houailou & 304 & n.d. \\
\hline \hline
\end{tabular}

MLVA, Multilocus variable number tandem repeat analysis; MLST, multilocus sequence typing, n.d., not determined. Italicized MLVA types are single locus variants of MLVA type 304 .

301, 302, 432), and three unrelated MLVA-4 types $(303,431,433)$. An additional three strains without MLST data were also MLVA-4 type 304. All six strains belonging to MLVA-4 type 304 originated 
from the same village of Houailou, with three of these cases diagnosed between 2006 and 2007. Furthermore, the four SLVs of MLVA-4 type 304 originated from neighboring villages (Ponerihouen and Poindimié). All but two of the other six MLVA-4 types (299, $303,305,431,433,434)$ originated from villages outside this region and those two were both ST292. Taken together, all 12 isolates from the region from Houailou to Poindimié were either MLVA-4 type 304 and/or MLST type 292. Additionally, a single ST292 isolate originated from outside of this region, in South Province. However, this patient's travel and medical history strongly suggested that she contracted melioidosis in Poindimié, North Province.

A comparison with representative strains of different geographical origin showed phylogenetic relatedness of New Caledonian isolates with isolates from Australia and PNG (Fig. 3). Out of the $16 \mathrm{New}$ Caledonian strains typed, all harboured the $B$. pseudomallei-like actin polymerization gene $\operatorname{bim} A_{\mathrm{Bp}}$.

\section{DISCUSSION}

Since the first human case was diagnosed in 1999, 19 melioidosis cases have been identified in New Caledonia, thereby extending the known endemic region of this infectious disease. All but one patient resided in the North Province and the travel and medical history of the remaining patient strongly suggested that she was infected in the North Province. This distribution might be slightly biased by access to hospital centres (Fig. 1); however, when facing severe infections, doctors in medical centres systematically send patients to the closest hospital, suggesting that this possible bias probably has a low effect on the true distribution. Another possible bias is the limited awareness of the New Caledonian medical community of melioidosis, suggesting an under-evaluation of the true incidence of this disease.

The observed restricted distribution of melioidosis to the North Province of New Caledonia suggests that there is a southern border for melioidosis within New Caledonia, although the reasons for this restriction are not fully understood. Interestingly, this geographical restriction resembles the pattern of melioidosis in PNG; the Balimo region has a high incidence for melioidosis, in contrast to other regions in PNG, where melioidosis incidence is low [7]. The restricted distribution of melioidosis cases in New Caledonia may reflect the lifestyle and cultural habits of individuals in the North Province, who mostly live as farmers in Melanesian tribes, with frequent contact with soil while growing yam, cassava and taro and with surface water in streams for showering, linen washing or fishing, all of which are less frequent in the more urbanized South Province. Farming and hunting activities also include frequent contact with swine (farmed and feral), feral deer and domestic horses. These activities have all formerly been identified as possible risk factors for melioidosis. Additionally, melioidosis infection most frequently occurs in the rainy season and the northeast coast is the wettest part of New Caledonia, another known risk factor for melioidosis. Whether the high metal content of the New Caledonian soil and the large-scale mining activity in this region also contribute to the survival and propagation of $B$. pseudomallei in the soil warrants consideration. Further environmental surveys for $B$. pseudomallei need to be conducted in this region [20-23].

The spatio-temporal distribution of cases in this study suggests that $B$. pseudomallei has been progressively moving from the far north towards the east coast since 2004. However, the MLST and MLVA typing results demonstrate that the strains responsible for melioidosis in the Houailou-Poindimié region did not originate from the strains isolated earlier from patients in the far north. Furthermore, our results suggest that one particular clonal group, characterized by MLST ST292 and MLVA type 304 and its SLVs, is predominant in this east coast region. The other cases outside this endemic region are mostly caused by other, unrelated B. pseudomallei strains.

All cases reported here were diagnosed by isolation of B. pseudomallei. Serology for melioidosis is not routinely used in New Caledonia and it is well recognized that background serology positivity in endemic regions makes serology of poor specificity for diagnosing melioidosis [6]. Nevertheless, our results suggest that a serological survey in the Houailou region and comparison with other locations throughout New Caledonia may be informative for directing further clinical and environmental sampling studies.

There is a wide range of clinical presentations of melioidosis, prompting its nickname as 'the great imitator'. One of the major differential diagnoses for pulmonary forms is tuberculosis which is also endemic in New Caledonia, as it is in PNG. The cases summarized in Table 1 were compared to Thai and Australian cohorts. Chronic infections were slightly more frequent in our cohort than in the Thai or Australian cohorts and skin disease was slightly more frequent than in the Thai cohort. In the Melanesian region, chronic infections 


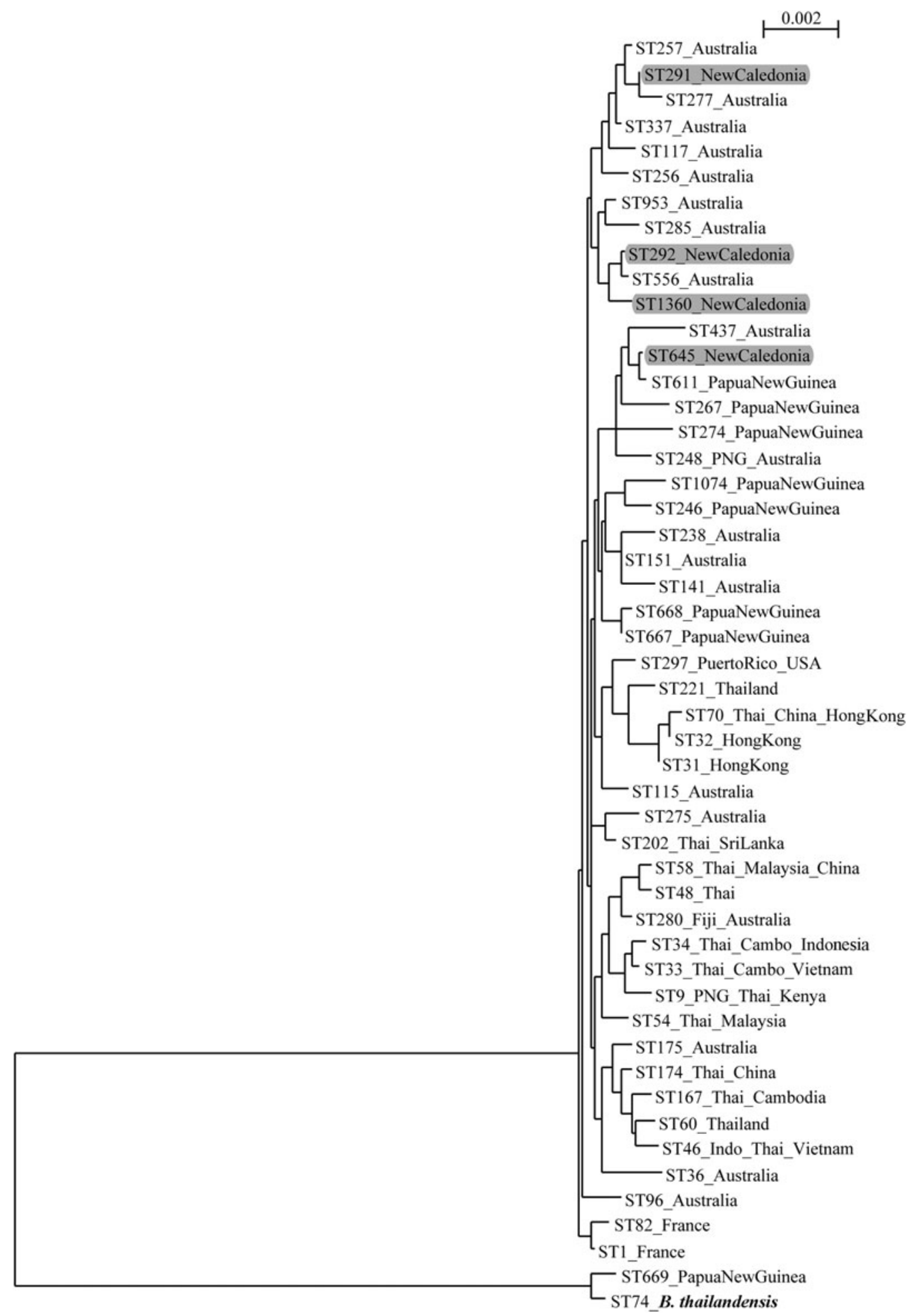

Fig. 3. Neighbour-Joining phylogeny of the New Caledonian B. pseudomallei and representative isolates as determined from concatenated multilocus sequence typing (MLST) alleles.

mimicking tuberculosis were also reported to be important [7]. Whether a particular cultural relationship with Western medicine defers visits to hospitals or medical centres and in turn contributes to this chronic clinical pattern in Melanesia deserves further consideration. Although bacteraemia was more frequent than in the Australian cohort, mortality was only $16 \%$. Pulmonary involvement was observed in $10(53 \%)$ 
patients, and meningitis was observed in a single case (Table 1). This finding is consistent with all New Caledonia strains tested thus far having the most pneumotropic and less neurotropic bim $A_{\mathrm{Bp}}$ genotype [15]. Within the Melanesian region, the pattern of melioidosis observed in the Balimo region of PNG for individuals with a median age of $<10$ years [7] still appears very specific and is not observed in New Caledonia.

The most frequently reported risk factors for melioidosis are diabetes, hazardous alcohol use and chronic lung disease [3]. Similar risk factors were found for most cases in our series. The treatment required for melioidosis is prolonged because of the high risk of relapse with shorter courses of therapy [24]. In our series, the median duration for intravenous therapy was 16.3 (range 3-24) days. Four patients did not receive the recommended antibiotic treatment but there was no documented relapse in any of the cases presented here. New Caledonia is endemic for leptospirosis, with severe disease well recognized $[25,26]$.

The similar environmental associations for both leptospirosis and melioidosis underlie the recognition that co-infections with both can occur, often with fatal outcomes [27]. To date there has been no co-infection diagnosed in New Caledonia, but heightened awareness of this possibility is important as therapy for each disease is different.

Our study reveals a heterogeneous distribution of melioidosis in New Caledonia. Interestingly, a specific region on the east coast with high incidence appears to be related to a specific $B$. pseudomallei clonal cluster, ST292. In contrast, B. pseudomallei from other regions of New Caledonia show considerable genetic diversity. Taken together, these results suggest that B. pseudomallei is widespread in the New Caledonian environment, particularly in the North Province and East Coast regions, with bacterial survival supported by favourable climatic conditions. Phylogenetic analysis of the MLST profiles of B. pseudomallei from New Caledonia shows a close relationship to isolates from Australia and PNG. It is likely that dispersal of $B$. pseudomallei occurred from Australia to New Caledonia, although the nature and timing of that dispersal remains to be elucidated [13,28]. One suggested possibility of $B$. pseudomallei transmission from Australia/PNG to New Caledonia is through migratory birds colonized with B. pseudomallei [29], although the trade of live horses and cattle may also be implicated. Another possibility is through the ancient movement of human populations at the time of the colonization of the insular Melanesia through
PNG, as hypothesized by Baker and colleagues [30]. Future studies utilizing whole genome sequence data are needed to provide more robust evaluation of these hypotheses.

In conclusion, this study provides the most comprehensive analysis of melioidosis cases in New Caledonia to date. The traditional lifestyle and habits of New Caledonians, particularly those in the North Province, provides an increased risk of $B$. pseudomallei transmission to these people and melioidosis awareness in these communities is needed. Both climate change and land perturbation through large-scale mining activities in New Caledonia may alter the presence and distribution of $B$. pseudomalle $i$ in this region, leading to a greater exposure risk and a potential increased incidence of melioidosis in the coming decades [28]. Environmental sampling of the New Caledonian environment is needed to determine $B$. pseudomallei prevalence and distribution across the country, and may identify hotspots for this bacterium. Finally, the findings from our study show that both inhabitants and healthcare providers in New Caledonia need to be aware of the risk factors for melioidosis, to both decrease infection rates and to enable rapid diagnosis and therapy, thereby minimizing morbidity and mortality from this emerging infectious disease.

\section{ACKNOWLEDGEMENTS}

We thank Direk Limmathurotsakul for fruitful scientific discussions and Vanessa Theobald for performing MLVA-4.

\section{DECLARATION OF INTEREST}

None.

\section{REFERENCES}

1. Wuthiekanun V, Smith MD, White NJ. Survival of Burkholderia pseudomallei in the absence of nutrients. Transactions of the Royal Society of Tropical Medicine and Hygiene 1995; 89: 491.

2. Currie BJ, et al. Endemic melioidosis in tropical northern Australia: a 10-year prospective study and review of the literature. Clinical Infectious Diseases 2000; 31: 981-986.

3. Cheng AC, Currie BJ. Melioidosis: epidemiology, pathophysiology, and management. Clinical Microbiology Reviews 2005; 18: 383-416.

4. Elliott JH, et al. Community-acquired pneumonia in northern Australia: low mortality in a tropical region 
using locally-developed treatment guidelines. International Journal of Infectious Diseases 2005; 9: 15-20.

5. Douglas MW, et al. Epidemiology of communityacquired and nosocomial bloodstream infections in tropical Australia: a 12-month prospective study. Tropical Medicine \& International Health 2004; 9: 795-804.

6. Wiersinga WJ, Currie BJ, Peacock SJ. Melioidosis. New England Journal of Medicine 2012; 367: 1035-1044.

7. Warner JM, et al. Melioidosis in a rural community of Western Province, Papua New Guinea. Transactions of the Royal Society of Tropical Medicine and Hygiene 2007; 101: 809-813.

8. Doker TJ, et al. Contact investigation of melioidosis cases reveals regional endemicity in Puerto Rico. Clinical Infectious Diseases 2015; 60: 243-250.

9. Currie BJ, et al. Neurological melioidosis. Acta Tropica 2000; 74: 145-151.

10. Domenech $\mathbf{J}$, et al. Animal health survey in New Caledonia [in French]. Revue d'Elevage et de Médecine Vétérinaire de Nouvelle-Calédonie 1984; 2: 1-8.

11. Poirier N. Melioidosis: a propos of a clinical case observed in New Caledonia: epidemiology, clinical and therapeutic aspects (MD dissertation) [in French]. Université de Rennes 1, 2001.

12. Estivals M, et al. Septicemia, bilateral community acquired pneumonia and empyema due to Burkholderia pseudomallei (melioidosis) with a favorable outcome following prolonged specific antibiotic therapy [in French]. Revue des Maladies Respiratoires 2008; 25: 319-322.

13. Le Hello S, et al. Melioidosis in New Caledonia. Emerging Infectious Diseases 2005; 11: 1607-1609.

14. Godoy D, et al. Multilocus sequence typing and evolutionary relationships among the causative agents of melioidosis and glanders, Burkholderia pseudomallei and Burkholderia mallei. Journal of Clinical Microbiology 2003; 41: 2068-2079.

15. Sarovich DS, et al. Variable virulence factors in Burkholderia pseudomallei (melioidosis) associated with human disease. PLoS ONE 2014; 9: e91682.

16. Currie BJ, et al. Identification of melioidosis outbreak by multilocus variable number tandem repeat analysis. Emerging Infectious Diseases 2009; 15: 169-174.

17. Hall TA. BioEdit: a user-friendly biological sequence alignment editor and analysis program for Windows 95/98/NT. Nucleic Acids Symposium Series 1999; 41: 95-98.
18. Galtier N, Gouy M, Gautier C. SEAVIEW and PHYLO_WIN: two graphic tools for sequence alignment and molecular phylogeny. Computer Applications in the Biosciences 1996; 12: 543-548.

19. Schweizer HP. Mechanisms of antibiotic resistance in Burkholderia pseudomallei: implications for treatment of melioidosis. Future Microbiology 2012; 7: 1389-1399.

20. Inglis TJ, Sagripanti JL. Environmental factors that affect the survival and persistence of Burkholderia pseudomallei. Applied and Environmental Microbiology 2006; 72: 6865-6875.

21. Palasatien S, et al. Soil physicochemical properties related to the presence of Burkholderia pseudomallei. Transactions of the Royal Society of Tropical Medicine and Hygiene 2008; 102 (Suppl. 1): S5-9.

22. Stoppel R, Schlegel HG. Nickel-resistant bacteria from anthropogenically nickel-polluted and naturally nickelpercolated ecosystems. Applied and Environmental Microbiology 1995; 61: 2276-2285.

23. Jones AL, DeShazer D, Woods DE. Identification and characterization of a two-component regulatory system involved in invasion of eukaryotic cells and heavy-metal resistance in Burkholderia pseudomallei. Infection and Immunity 1997; 65: 4972-4977.

24. Pitman MC, et al. Intravenous therapy duration and outcomes in melioidosis: a new treatment paradigm. PLoS Neglected Tropical Diseases 2015; 9: e0003586.

25. Tubiana S, et al. Risk factors and predictors of severe leptospirosis in New Caledonia. PLoS Neglected Tropical Diseases 2013; 7: e1991.

26. Goarant C, et al. Outbreak of leptospirosis in New Caledonia: diagnosis issues and burden of disease. Tropical Medicine \& International Health 2009; 14: 926-929.

27. Hin HS, et al. Fatal co-infection-melioidosis and leptospirosis. American Journal of Tropical Medicine and Hygiene 2012; 87: 737-740.

28. Currie BJ. Melioidosis: evolving concepts in epidemiology, pathogenesis, and treatment. Seminars in Respiratory and Critical Care Medicine 2015; 36: 111-125.

29. Hampton V, et al. Melioidosis in birds and Burkholderia pseudomallei dispersal, Australia. Emerging Infectious Diseases 2011; 17: 1310-1312.

30. Baker A, et al. Molecular phylogeny of Burkholderia pseudomallei from a remote region of Papua New Guinea. PLoS ONE 2011; 6: e18343. 